\title{
Structural Racism and HIV Risk: Low African American Male to Female Sex Ratio and HIV Diagnosis Rates by State
}

\author{
Leonard Anang Sowah, MBChB, MPH, FACP ${ }^{1,2^{*}}$ and Brittany Yerkes, $P A, M S c^{3}$ \\ ${ }^{1}$ Adjunct Assistant Professor, University of Maryland School of Medicine, USA \\ ${ }^{2}$ National Institute of Allergy and Infectious Diseases, Maryland, USA \\ ${ }^{3}$ University of Maryland Eastern Shore, Easton Maryland, USA
}

*Corresponding author: Leonard Anang Sowah, MBChB, MPH, FACP, Adjunct Assistant Professor, University of Maryland School of Medicine, 725 W. Lombard Street, Baltimore, USA

\begin{abstract}
HIV diagnosis rates in the US differ significantly by race and ethnicity. There are also geographic differences in HIV diagnosis rates across US states and regions. The determinants of these differences are not always easy to disentangle. Some believe structural racism may contribute to the differences in HIV diagnosis by race. This may also explain state and regional variations in HIV diagnosis. Using data from the 2010 US census and 2010 HIV surveillance data from CDC we did a test to determine if there is a correlation between state level African American sex ratio and HIV diagnosis rates.

The Pearson's correlation coefficient between sex ratio by state and HIV diagnosis rates was $(r=-0.61, P<0.0001)$ suggesting moderate correlation between the two variables. Our study suggests there may be proximate factors linking sex-ratio in African American communities to state level HIV diagnosis rates. State and public health institutions must therefore consider these structural factors in their HIV prevention efforts.
\end{abstract}

\section{What do we already know about this topic?}

Prior research does suggest that low sex-ratio secondary to high rates of incarceration and violence in African American communities could impact risk of sexually transmitted infections.

\section{How does your research contribute to the field?}

This research suggests that skewed sex ratios could potentially be influencing population level HIV risk across communities.
What are your research's implications towards theory, practice, or policy?

More studies are required to better understand the drivers of this relationship. If this relationship found to be consistent, policy makers and public health experts in HIV prevention must consider sex ratio as a potential intervention target for HIV prevention.

\section{Introduction}

In the United States HIV diagnosis rates differs significantly by race. A recent CDC surveillance report suggests that HIV diagnosis rates is almost 8 times higher in African American men compared to whites and 13 times in African American women compared to white women. Geographically in the US HIV diagnosis rates also differ by state, with states in the Northeast and the South having higher rates compared to other regions [1]. With these obvious significant differences in HIV transmission rates many have tried to identify the determinants of HIV transmission rates by race. The reasons for this disparity in HIV transmission risk by race are not clearly understood but there are many theories. On looking at these variations in sex ratio within African American communities and HIV diagnosis rates we came up with a hypothesis on differences in HIV diagnosis rates by states across the US. Our hypothesis which attempts to find an association between discrimination, structural racism and HIV diagnosis rates was based on prior research on population level sex ratio and diagnosis of 
sexually transmitted infections (STIs). Community level, low male to female sex ratios has social and community consequences, especially pertaining to STIs. This has been demonstrated in studies focusing on communities impacted by high rates of male incarceration secondary to implicit bias and structural racism, resulting in low community sex ratios [2].

We postulated that HIV transmission risk and thus diagnosis rates by state will be negatively associated with state level sex ratios. Since state level African American sex-ratios were more variable across states and regions we used that population characteristic as our determinant. To test out this hypothesis we investigated the correlation between state level African American sex ratios and HIV diagnosis rates. The theory and ideas underlying our hypothesis is shown in the conceptual framework shown in Figure 1.

\section{Methods}

The study utilized US 2010 Census data and 2010 state level HIV diagnosis data, as reported on AIDSVu. org. State sex-ratio data was calculated to include all individuals' ages 15-64 years who self-identified as African American or reported black, African, or Caribbean heritage on census forms. Individuals who selected multiple races were included if they selected African or African American as one of their selected racial descriptions. Pearson's correlation coefficients were calculated comparing HIV diagnosis rates by state and region to determine the strength and nature of the association.

To determine the validity of the correlation coefficients for the identified demographic variables we utilized the rule of thumb [3]. Any Pearson's correlation coefficient ( $r$ ) that failed to meet this rule, $|r| \geq 2 / V_{n}$ where $\mathrm{n}=$ sample size, was considered not statistically significant. All analysis was done using Stata version 10 software; StataCorp. 2007. Stata Statistical Software: Release 10. College Station, TX: StataCorp LP.

\section{Results}

\section{Sex ratio and HIV diagnosis rates by State}

Sex ratios for the African American population ages 15-64 year differed by state and ranged from $80.2 \%$ in Vermont to a high of $163.5 \%$ in Wyoming. State level HIV diagnosis rates for the year 2010 ranged from 2 per 100,000 in states like Montana and North Dakota to a high of 38 per 100,000 persons in Maryland. States with sex ratios less than $90 \%$ were more likely to have higher HIV diagnosis rates. With the exception of Vermont with a rate of 4 per 100,000 persons the HIV diagnosis rates of these states ranged from 15 per 100,000 persons in Illinois and Tennessee with sex ratios of $87.6 \%$ and $88.6 \%$ respectively to 38 per 100,000 persons in Maryland with a sex ratio of $85.7 \%$. The other low sex ratio states are Alabama sex ratio of $86.4 \%$ with HIV diagnosis rate of 17 per 100,000 , Delaware with sex ratio of $87.7 \%$ and also

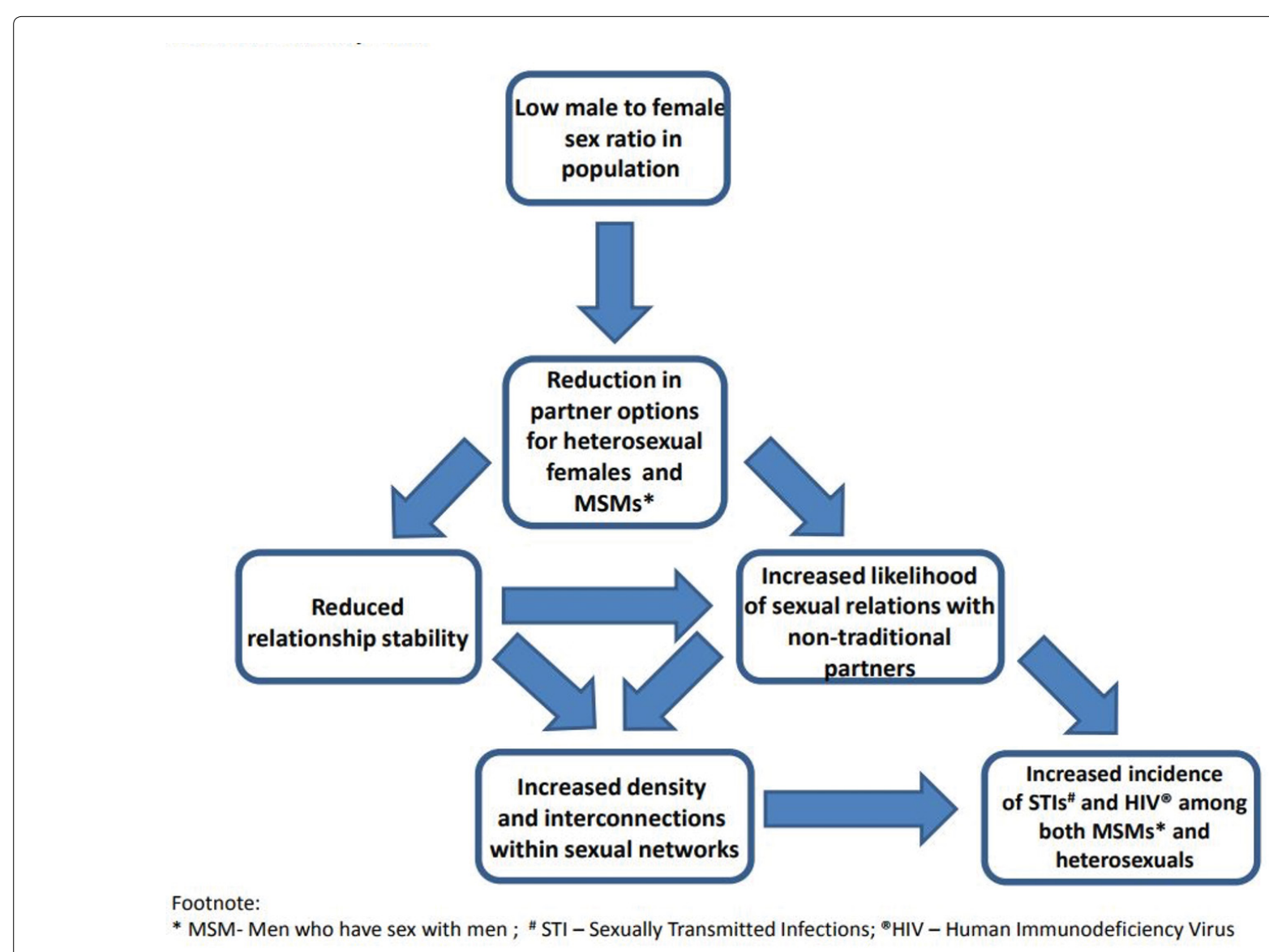

Figure 1: Conceptual framework explaining the relationship between sex ratio and HIV diagnosis rates. 


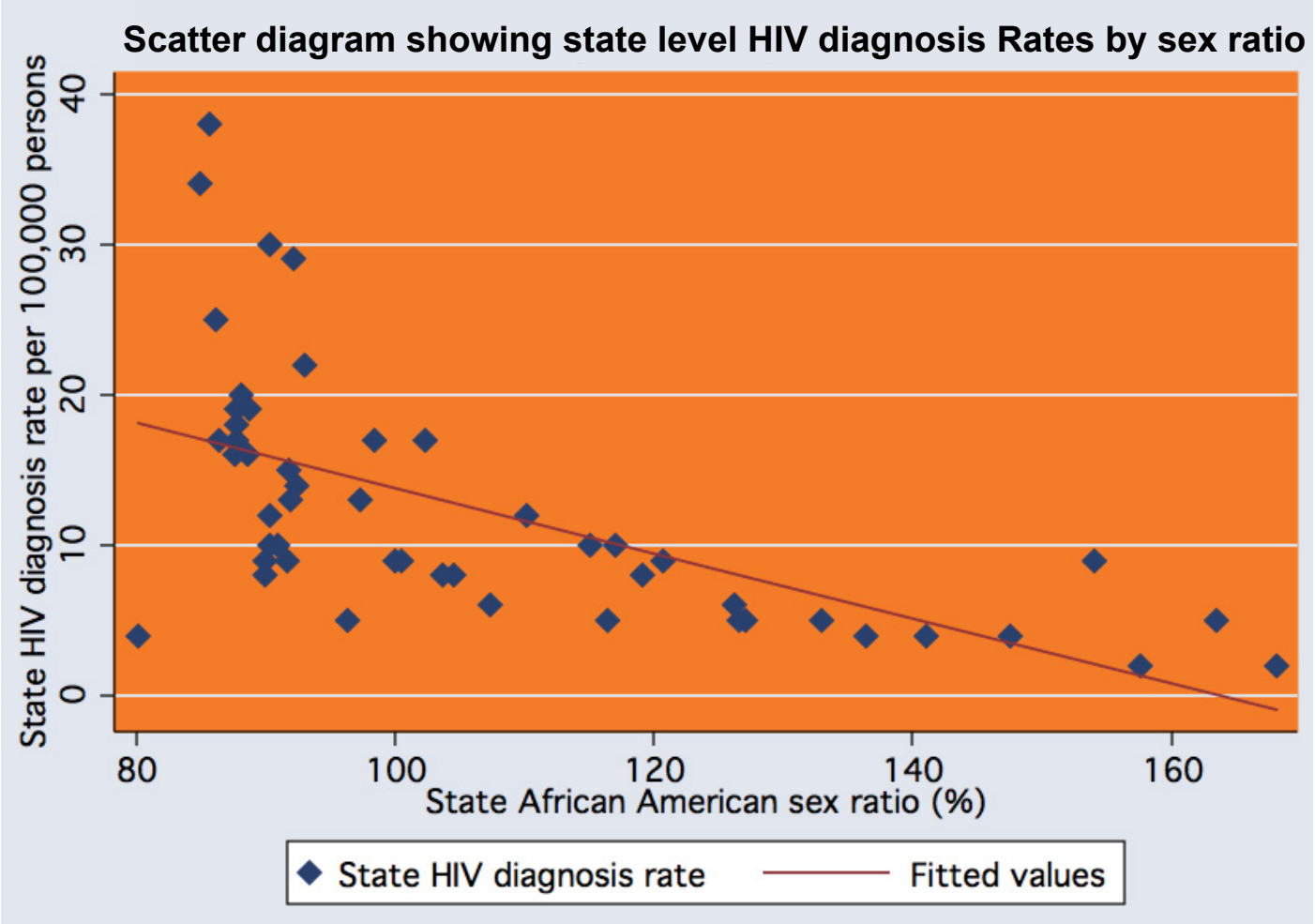

Figure 2: Figure showing scatter plot of HIV African American sex-ratios and state level diagnosis rates for the year 2010. The plot shows good correlation with a coefficient of 0.61 , with outliers like Vermont in the lower right with HIV diagnosis rate of 4 per 100,000 and a sex-ratio of 80.2 . In the upper left Maryland with sex-ratio $85.7 \%$ and diagnosis rate of 38 per 100,000 and Georgia $84.9 \%$ and a diagnosis rate of 34 per 100,000 .

Table 1: Showing Pearson's Correlation Coefficients of and Coefficient of Determination between HIV diagnosis rates and African American community sex ratios by US Regions.

\begin{tabular}{|c|c|c|c|c|c|c|}
\hline & Region & $\begin{array}{l}\text { Sample } \\
\text { Size }\end{array}$ & $\begin{array}{l}\text { Pearson } \\
\text { Correlation } \\
\text { Coefficient }(r)\end{array}$ & $\begin{array}{l}\text { Coefficient of } \\
\text { determination }\left(r^{2}\right) \\
(\%)\end{array}$ & p-value & $\begin{array}{l}\text { Critical } \\
\text { values } \\
|r| \geq 2 / \sqrt{ } n\end{array}$ \\
\hline \multirow{6}{*}{$\begin{array}{l}\text { Correlation of } \\
\text { HIV diagnosis } \\
\text { rates with sex } \\
\text { ratio }\end{array}$} & All States \& DC & 51 & -0.35 & 12.5 & $<0.01$ & 0.28 \\
\hline & All States & 50 & -0.61 & 37.21 & $<0.0001$ & 0.28 \\
\hline & Northeast & 9 & -0.49 & 24.01 & 0.5 & 0.63 \\
\hline & Midwest & 12 & -0.71 & 50.41 & 0.01 & 0.55 \\
\hline & West & 13 & -0.84 & 70.56 & 0.0003 & 0.58 \\
\hline & South & 16 & -0.59 & 34.81 & 0.02 & 0.52 \\
\hline
\end{tabular}

All significantly p-values for the relationship between sex ratio and state level HIV diagnosis rates are in bold text.

with a diagnosis rate of 17 per 100,000, North Carolina with a sex ratio $87.7 \%$ with HIV diagnosis rate of 18 per 100,000 , New Jersey with sex ratio of $88.8 \%$ and diagnosis rate of 19 per 100,000 , Mississippi with sex ratio $87.8 \%$ and a diagnosis rate of 19 per 100,000 , South Carolina with a sex ratio of $88.1 \%$ and diagnosis rate of 20 per 100,000 , New York with a sex ratio of $86.1 \%$ and a sex ratio of 25 per 100,000 persons and Georgia with sex ratio of $84.9 \%$ and a diagnosis rate of 34 per 100,000 persons. The plot shows a negative correlation between HIV diagnosis rates and sex ratio by state (Figure 2). The Pearson's correlation coefficient comparing sex ratio to HIV diagnosis rate by state was significant $(r=-0.61$, $\mathrm{P}<0.0001$ ) suggesting a moderately strong correlation between the two variables (See Table 1).
In regional subgroup analysis this correlation was significant for all regions of the US with the exception of the Northeast. The strongest correlation between sex ratio and HIV diagnosis rates was in the West with a Pearson's correlation coefficient $(r=-0.84, P<0.0003)$ (Table 1).

\section{Discussion}

Our research revealed a moderately strong correlation between low sex ratio and HIV diagnosis rates by state. Prior studies have focused on the relationship between incarceration rates and diagnoses of HIV and STIs, one such study showing a significant correlation between chlamydia rates and high rates of incarceration [4]. Our analysis, whilst not proving any 
form of causality, does suggest there may be proximate factors linking these two variables. There was a trend for low sex ratio states in the South and the Northeast and higher sex ratios in the West and Mountain states. This trend of low sex ratios in these states may be a result of structural and systemic racism driving the male African America populations away from these regions or into the criminal justice system. Studies by social scientists suggest that high black male incarceration rates secondary to systemic racism may account for this trend [5]. Researchers and public health authorities attempting to address the HIV epidemic would need to study community sex ratios and HIV diagnosis rates more closely. This clear association between state level HIV diagnosis rates and sex-ratio suggests that public health and legislative authorities must pay closer attention to interventions targeting structural factors driving HIV transmissions.

\section{Conflict of Interest}

Authors reports no conflict of interest related to this work.

\section{Disclaimer}

The information presented in this paper represent the views and opinions of the authors and must not be taken as representing the opinions or views of any organizations that they work for or represent in other ways.

\section{Funding}

No funding support for this research.

\section{References}

1. Centers for Disease Control and Prevention (2020) Estimated HIV incidence and prevalence in the United States, 2014-2018. HIV Surveillance Supplemental Report 2020: 25.

2. Green TC, Pouget ER, Harrington M, Taxman FS, Rhodes AG, et al. (2012) Limiting options: Sex ratios, incarceration rates and sexual risk behavior among people on probation and parole. Sexually transmitted diseases 39: 424-430.

3. Krehbiel TC (2004) Correlation coefficient rule of thumb. Decision Sciences Journal of Innovative Education 2: 97100.

4. Dauria EF, Elifson K, Arriola KJ, Wingood G, Cooper HL (2015) Male incarceration rates and rates of sexually transmitted infections: Results from a Longitudinal Analysis in a South-Eastern US City. Sexually transmitted diseases 42: 324-328.

5. Eriksson K (2019) Moving North and into jail? The great migration and black incarceration. Journal of Economic Behavior \& Organization 159: 526-538. 